\title{
Characterising refractive index dispersion in chalcogenide glasses
}

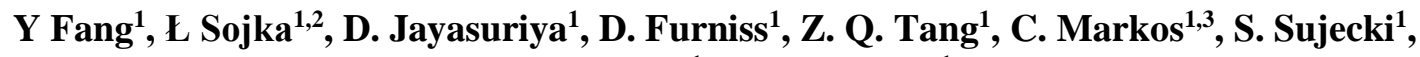 \\ A. B. Seddon ${ }^{1}$, T. M. Benson ${ }^{1}$ \\ ${ }^{1}$ Mid-Infrared Photonics Group, George Green Institute for Electromagnetics Research, University of \\ Nottingham, University Park, NG7 2RD Nottingham, UK, Tel: (+44115) 95155889, Fax: (+44115) 9515616 \\ e-mail: Trevor.Benson@nottingham.ac.uk \\ ${ }^{2}$ Institute of Telecommunication, Teleinformatics \& Acoustics, Wroclaw University of Technology, Wybrzeze \\ Wyspianskiego 27, 50-370 Wroclaw, Poland \\ ${ }^{3}$ DTU Fotonik, Dept. of Photonics Engineering, Technical University of Denmark, Ørsteds Plads 343, 2800 \\ Kongens, Lyngby, Denmark
}

\begin{abstract}
Much effort has been devoted to the study of glasses that contain the chalcogen elements (sulfur, selenium and tellurium) for photonics' applications out to MIR wavelengths. In this paper we describe some techniques for determining the refractive index dispersion characteristics of these glasses. Knowledge of material dispersion is critical in delivering step-index fibres including with high numerical aperture for mid-infrared supercontinuum generation.
\end{abstract}

Keywords: chalcogenide glasses, refractive index, dispersion.

\section{INTRODUCTION}

Chalcogenide glasses, containing the chalcogen elements sulfur, selenium and tellurium, have many attributes that make them attractive for photonics applications at the mid-infrared (MIR) wavelengths of 3-25 $\mu$ m [1-4]. Critical for many of these applications, for example medical diagnostics, power delivery, microscopy and sensing, is the potential for low loss fibre fabrication [5-12] and hence thermal glass stability against devitrification. Many glass compositions are available, which provides the opportunity and versatility to realise glasses with a wide and continuous range of physical properties, including refractive index. Allowing thermal matching, e.g. of glass transition temperature and viscosity/temperature behaviour, of the core and cladding materials helps in realising fibres of good interface quality [13].

Refractive index, and very often its behaviour over a range of wavelengths and temperatures, is one of the key parameters that influence the design of optical components. Here we concentrate on wavelength dispersion at ambient temperature. In previous work, we reported on the measurement at ambient temperature of the linear refractive index dispersion of two chalcogenide glass compositions over the wavelength range $0.4 \mu \mathrm{m}$ to $33 \mu \mathrm{m}$ by means of spectroscopic ellipsometry [14]. The fitting of the data to a suitable model is extremely useful for describing the glasses within electromagnetic simulation tools. A two-term Sellmeier equation was shown sufficient to describe the refractive index dispersion over the wavelength range for which the experimentally determined extinction coefficient was unimportant [14]. The two-term Sellmeier model fitted the data points measured by ellipsometry to better than $0.1 \%$. However, the accuracy of this method is limited by glass surface effects. The strength of the ellipsometry, its extreme surface sensitivity, is also its potential weakness [15]. A very thin contamination layer on a thin film could potential yield different optical constants for the thin film. In [16] we demonstrated mid-infrared supercontinuum generation in a high numerical aperture chalcogenide glass step-index fibre formed using the two glasses studied in [14], and verified calculations of fibre dispersion based upon the two measured material refractive indices using spectral-domain interferometry.

The prism coupling technique has also been utilised to measure the refractive index of chalcogenide glasses in bulk and thin film form in the near- and MIR spectral regions. A commercial system (Metricon model 2010) has been modified with additional laser sources, detectors, and a new GaP prism to allow the measurement of refractive index dispersion over the $1.5-10.6 \mu \mathrm{m}$ range. The instrumental error was found to be \pm 0.001 refractive index units across the entire wavelength region examined. Carlie et al. [17] measured the refractive index of $\mathrm{As}_{2} \mathrm{Se}_{3}$ bulk glasses and compared the refractive indices of as-deposited and annealed $\mathrm{As}_{2} \mathrm{Se}_{3}$ films with those of the parent bulk glasses. The measured refractive index of the as-deposited films was found to be significantly lower than that of the bulk glass to subsequently increase to a value near that of the bulk upon annealing near the 
glass transition temperature of the parent bulk glass. This result shows the importance of the thermal history of the glass on its physical structure and consequently on its physical properties such as density and refractive index. Qiao et al. [18] used this method to measure the refractive indices of several chalcogenide glass families over the $1.5-10.6 \mu \mathrm{m}$ range with an accuracy of $\pm 1 \times 10^{-3}$.

In this paper, we report our progress towards adapting two other well-known methods in order to determine the optical properties of chalcogenide glasses in the MIR region. The first is the minimum deviation method [19] which determines refractive index from the minimum deviation angle of a ray passing through a prism. The second is the method of Swanepoel [20] which was originally presented to determine the refractive index and thickness of thin films in the transparent region from wavelength values at maxima and minima of the transmission interference fringes only. This entails measuring two transmission spectra, one at normal incidence and another at oblique incidence. This method was used by Corrales et al. to measure the refractive index of an $\mathrm{As}_{40} \mathrm{Se}_{60}$ thin film of approximate thickness in 600-2000 $\mathrm{nm}$ wavelength region [21] and the method yielded values for refractive index and average thin film thickness to an accuracy of better than $3 \%$.

\section{MINIMUM DEVIATION METHOD}

When a laser beam travels through a prism with a certain incident angle, the minimum deviation position will be achieved which makes the sum of the two deflections a minimum. Applying Snell's law and using geometric optics, an expression for refractive index $\mathrm{n}$ can be obtained as

$$
n=\frac{\sin [(\alpha+\theta) / 2]}{\sin (\alpha / 2)}
$$

where $\alpha$ is the apex angle of prism, and $\theta$ is the minimum deviation angle. As with the prism coupling technique the extension of this method towards the MIR has become technologically possible in recent years by the development of new light sources such as MIR quantum cascade lasers and MIR supercontinuum sources, and new photovoltaic detectors covering the wavelength range up to $12 \mu \mathrm{m}$

The chalcogenide glass sample preparation procedure was as follows:

1. An as-synthesised, as-annealed chalcogenide glass rod [1] of $\sim 10 \mathrm{~mm}$ diameter was placed on a home-made metal holder and a saw (Supplier: ISOMET) and a Buehler diamond wafering blade were used to cut thought the glass rod in order to make a $10-15 \mathrm{~mm}$ long glass rod.

2. The glass rod was placed in a plastic holder with some silicon spray (Supplier: Force) on its internal surface. Monomer epoxy liquid was poured on to the rod to completely immerse it. The holder with the sample was left for 24 hours for polymerisation to occur.

3. The polymer-encapsulated glass rod sample was taken out of the holder and hot mounted on a home-made metal holder at $\sim 80{ }^{\circ} \mathrm{C}$ using wax (Buehler, Coventry, UK), as shown in Figure 1a. The holder with the sample was placed on the Buehler saw and was cut through at the dotted line as shown on Figure 1a. After cutting, the sample looks like the schematic shown in Figure 1b. Then the chalcogenide glass sample was ground and polished to a $1 \mu \mathrm{m}$ finish.

4. The one-face-polished sample was removed from the holder and mounted on a second holder whose top had been machined to the desired prism angle (Fig. 1c). A cut was made, as indicated by the dotted line in Fig.1c, using the saw, and grinding and polishing then performed to a $1 \mu \mathrm{m}$ finish. The final prism with the desired apex angle is shown schematically in Fig. 1d. An increase in the apex angle reduces the standard deviation of the results. However, if the apex angle is too large, the beam is unable to pass through the prism. A $20^{\circ}$ prism apex angle is a good choice for chalcogenide glasses (for refractive indices 2.2 to 3.5 ).

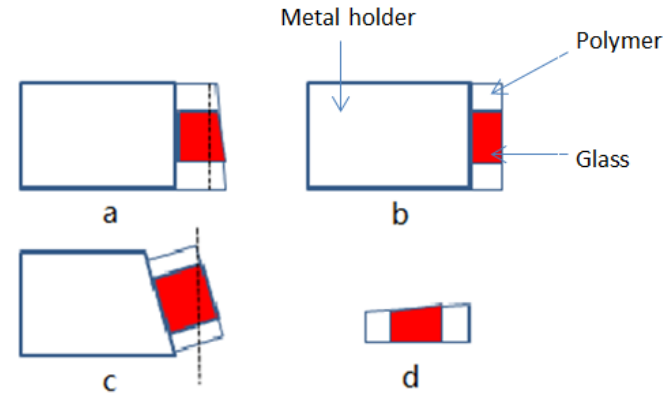

Figure 1 Schematic diagrams showing the prism preparation procedure 
For measurement of the apex angle $\alpha$, a laser beam was launched on to the surface of the chalcogenide glass prism. The prism was adjusted to let the incident beam reflect back along its own path at a recorded angle of $\alpha_{1}$. The prism was rotated to let the other surface reflect the beam back along its own path at some angle $\alpha_{2}$. The apex angle $\alpha$ was then found as $\alpha=\left(\alpha_{1}-\alpha_{2}-180\right)^{\circ}$. The prism was rotated continuously in the same clockwise direction and the light ray was observed to rotate in the same direction as the prism up to a certain point, at which the light beam would change its direction of travel. This is the minimum deviation position. Retroreflection, using a mirror placed on a second turntable, was used to determine the minimum deviation angle.

The standard deviation in the refractive index measurement was found to be 0.0034 for a $10^{\circ}$ apex angle and 0.0008 for a $20^{\circ}$ apex angle. This measurement accuracy requires a good surface finish, and can be improved by using a better collimated beam and increasing the optical path from the prism to the detector used in determining the minimum deviation position. The advantage of the minimum deviation method is that it can provide highly accurate and reproducible results at available source wavelengths. Moreover, the refractive index measurement is less sensitive than ellipsometry to the effect of an oxide layer on the glass surface. However, the minimum deviation technique requires intensive and time-consuming sample preparation.

\section{EXTENSIONS TO THE METHOD OF SWANEPOEL.}

The method of Swanepoel [20] necessitates obtaining two interference transmission spectra through a thin film, one at normal incidence and another at oblique incidence. In his paper the method yielded the values of thin film refractive index and thickness to an accuracy of better than 1\%. The method, as reported in [20] used a Cauchy equation to determine the refractive index of the thin film. According to [14], the Cauchy model does not encompass the entire glass transparent window and so in the present work the Cauchy dispersion equation used in [20] was changed to a two-term Sellmeier dispersion equation to extend the potential use of the method for determining the optical properties of chalcogenide thin film in the MIR region. The two methods gave consistent refractive index results from the experimental data of [21] over the 600-2000 nm wavelength region.

In support of this work, a hot pressing technique has been developed for the fabrication of free standing chalcogenide glass samples of thickness around $25 \mu \mathrm{m}$ and whose transmission spectra can be measured using Fourier Transform Infrared (FTIR) spectrometry. The hot press consists of two flat aluminium plates of diameter $\sim 150 \mathrm{~mm}$ inside a vacuum chamber which was evacuated to $10^{-4} \mathrm{~Pa}$ by means of a rotary pump. The temperature of each plate was controlled and was monitored by a thermocouple. The base plate was allowed to move up-anddown and the load applied between the two plates was monitored during hot pressing. A height readout gave the changing height of the base plate up to $0.01 \mathrm{~mm}$ accuracy. Lengths of chalcogenide glass monostructured optical fibre of about $20 \mathrm{~mm}$, and with a diameter of $\sim 240 \mu \mathrm{m}$, were pressed in order to obtain the chalcogenide glass thin films. Fibres were placed on the base tungsten carbide plate, which rested on the aluminium base plate of the hot press. The fibres were then covered with the second tungsten carbide plate. A stainless steel shim (26 $\mu \mathrm{m}$ thick) (Hollinbrow Ltd) was placed around the fibres, as shown in Fig. 2a. A typical pressing schedule for $\mathrm{As}_{40} \mathrm{Se}_{60}$ (atomic \% (at\%)) is shown in Fig. 2 b.

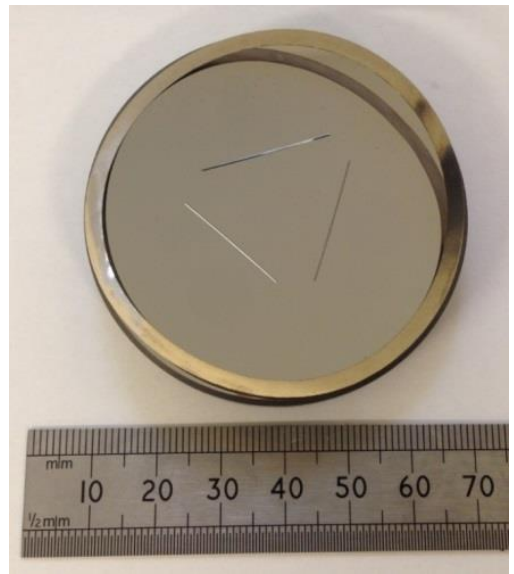

(a)

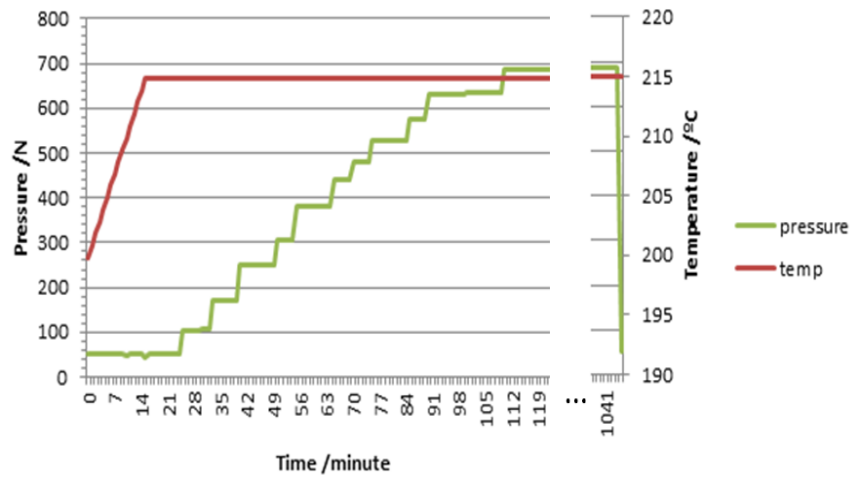

(b)

Figure 2 (a) Three $\mathrm{As}_{40} \mathrm{Se}_{60}$ (at\%) fibres surrounded by a $25 \mu \mathrm{m}$ thick stainless steel shim placed on a tungsten carbide plate ready for hot pressing. (b) Typical pressing schedule for AsSe. 
After the hot pressing, the pressed samples were annealed by cooling from the pressing temperature to the glass transition temperature, $\mathrm{T}_{\mathrm{g}}\left(180{ }^{\circ} \mathrm{C}\right.$ for $\left.\mathrm{As}_{40} \mathrm{Se}_{60}(\mathrm{at} \%)\right) @ 100{ }^{\circ} \mathrm{C} / \mathrm{h}$, dwelling at $\mathrm{T}_{\mathrm{g}}$ for $1 \mathrm{~h}$ and then cooling down naturally with the equipment to room temperature, which typically took over 8 hours.

Figure 3 shows a transmission spectrum recorded using a Bruker IFS 66v/s FTIR for a pressed $\mathrm{As}_{40} \mathrm{Se}_{60}($ at $\%$ ) thin film sample at normal incidence (blue curve) and at an angle of incidence of $30^{\circ}$ (red curve). The thickness was found to be $29.0 \pm 0.3 \mu \mathrm{m}$ from the Swanepoel method. The beam in the FTIR is not fully collimated and this gives scope for further improvement of the method in the future.

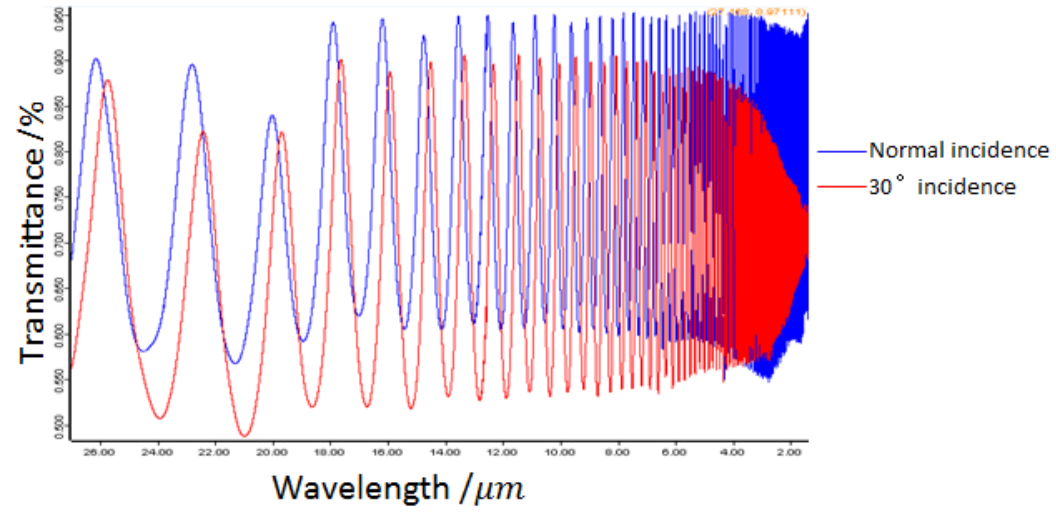

Figure 3. Transmission spectra for a $29 \mu \mathrm{m}$ thick pressed $\mathrm{As}_{40} \mathrm{Se}_{60}$ (at\%) sample at normal incidence (blue curve) and at ancidence angle of $30^{\circ}$ (red curve).

Fig. 4a shows a Sellmeier fit to the resultant refractive index results for this sample, and compares them with the results presented for the same nominal glass composition in [14]. Fig. 4b shows similar Sellmeier fits obtained from a set of $\mathrm{As}_{40} \mathrm{Se}_{60}$ (at\%) samples pressed under the same conditions but on different occasions. These refractive index results show agreement to within $2 \%$, but are consistently higher than those reported in [14]. The Swanepoel method is sensitive enough to pick up in the raw refractive index data the effect of absorption at $20.8 \mu \mathrm{m}$, associated with a second overtone of a fundamental stretching band [22, 14].
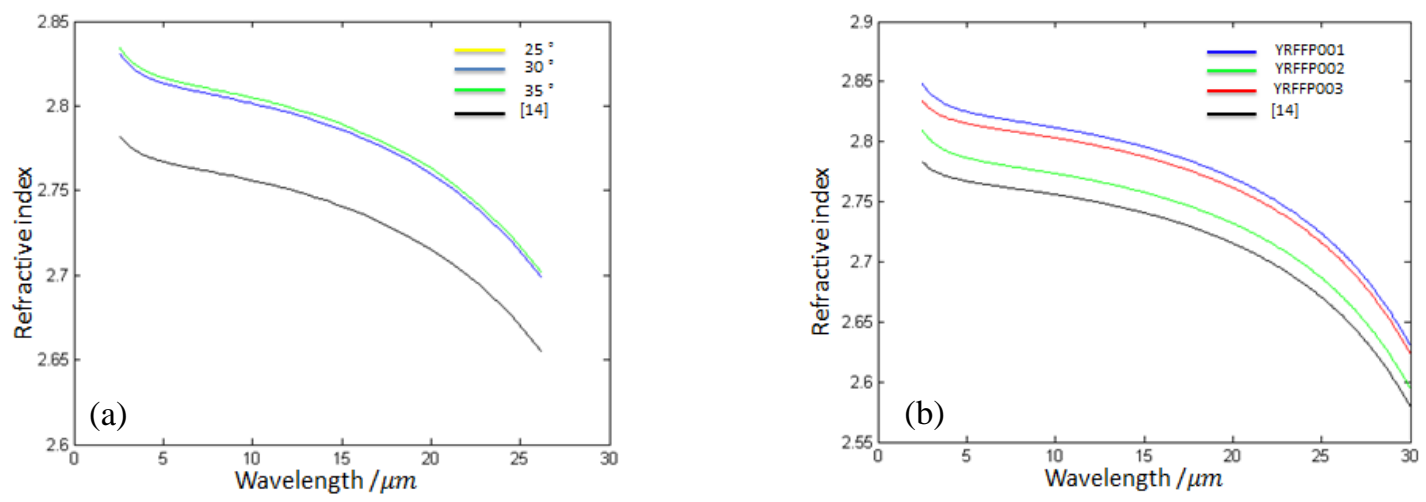

Figure 4 (a) Refractive index data for $\mathrm{As}_{40} \mathrm{Se}_{60}$ (at\%) fitted by a two-term Sellmeier equation obtained using the Swanepoel method for the sample shown in Figure 4 obtained with different incident angles. (b) Similar results obtained from a set of samples pressed on different occasions. Both plots also present the data from [14] for comparison purposes.

\section{CONCLUSIONS}

In this paper some techniques for measuring the refractive index dispersion characteristics of chalcogenide glasses out to MIR wavelengths have been described. Refractive index, and its dispersion, is a fundamental property in the design of optical fibres, yet it can not only be affected by compositional changes but by the thermal history of the glass. Techniques such as ellipsometry and prism coupling are now well established, but ellipsometry is sensitive to surface effects. The minimum deviation method is accurate, but needs specialist timeconsuming sample preparation. The Swanepoel method [20] determines refractive index from interference transmission spectra measured through a thin film, one at normal incidence and another at oblique incidence. The preparation of stand-alone thin films for this purpose by pressing fibres is promising. The present extension to the use of a two-term Sellmeier equation improves the fitting to experimental data out to MIR wavelengths. 


\section{REFERENCES}

1. A. B. Seddon, "Chalcogenide glasses: A review of their preparation, properties and applications," J. NonCryst. Solids 184(0), 44-50 (1995).

2. A. Zakery and S. Elliott, "Optical properties and applications of chalcogenide glasses: A review," J. NonCryst. Solids 330(1), 1-12 (2003).

3. B. J. Eggleton, "Chalcogenide photonics: Fabrication, devices and applications introduction," Opt. Express 18(25), 26632-26634 (2010).

4. B. J. Eggleton, B. Luther-Davies and K. Richardson, "Chalcogenide photonics", Nature Photon. 5, 141148 (2011).

5. J. S. Sanghera and I. D. Aggarwal, "Active and passive chalcogenide glass optical fibers for IR applications: A review,” J. Non-Cryst. Solids 256-257(0), 6-16 (1999).

6. J.-L. Adam, L. Calvez, J. Trolès and V. Nazabal, "Chalcogenide glasses for infrared photonics,” Int. J. Appl. Glas. Sci., vol. 6, no. 3, pp. 287-294 (2015)

7. V. S. Shiryaev and M. F. Churbanov, "Trends and prospects for development of chalcogenide fibers for mid-infrared transmission", J. Non-Cryst. Solids 377, 225-230 (2013).

8. M. Churbanov, G. Snopatin, V. Shiryaev, V. Plotnichenko and E. Dianov, "Recent advances in preparation of high-purity glasses based on arsenic chalcogenides for fiber optics," J. Non-Cryst. Solids 357(11), 2352-2357 (2011).

9. V. Q. Nguyen, J. S. Sanghera, P. Pureza, F. H. Kung and I. D. Aggarwal, "Fabrication of arsenic selenide optical fiber with low hydrogen impurities," J. Am. Ceram. Soc. 85(11), 2849-2851 (2002).

10. Z. Tang, D. Furniss, M. Fay, N. C. Neate, Y. Cheng, E. Barney, L. Sojka, S. Sujecki, T. M. Benson and A. B. Seddon, "First identification of rare-earth oxide nucleation in chalcogenide glasses and implications for fabrication of mid-infrared active fibers," J. Am. Ceram. Soc. 97(2), 432-441 (2014).

11. Z. Tang, V. S. Shiryaev, D. Furniss, L. Sojka, S. Sujecki, T. M. Benson, A. B. Seddon and M. F. Churbanov, "Low loss Ge-As-Se chalcogenide glass fiber, fabricated using extruded preform, for midinfrared photonics." Optical Materials Express 5(8), 1722-1737 (2015).

12. G. S. Athanasiou, J. Ernst, D. Furniss, T. M. Benson, J. Chauhan, J. Middleton, C. Parmenter, M. Fay, N. Neate, V. Shiryaev, M. F. Churbanov and A. B. Seddon, "Toward mid-Infrared, subdiffraction, spectralmapping of human cells and tissue: SNIM (Scanning Near-Field Infrared Microscopy) tip fabrication." Journal of Lightwave Technology 34(4), 1212-1219 (2016).

13. V. Moizan, V. Nazabal, J. Troles, P. Houizot, J-L. Adam, J. L. Doualan, R. Moncorgé, F. Smektala, G. Gadret, S. Pitois and G. Canat, " $\mathrm{Er}^{3+}$-doped GeGaSbS glasses for MIR fibre laser application: Synthesis and rare earth spectroscopy," Opt. Mater. 31(1), 39-46 (2008).

14. H.G. Dantanarayana, N. Abdel-Moneim, Z. Tang, L. Sojka, S. Sujecki, D. Furniss, A.B. Seddon, I. Kubat, O. Bang and T. M. Benson, "Refractive index dispersion of chalcogenide glasses for ultra-high numericalaperture fiber for mid-infrared supercontinuum generation”, Optical Materials Express, 4(7), 1444-1455 (2014.)

15. D. Poelman and P. Smet, "Methods for the determination of the optical constants of thin films from single transmission measurements: A critical review", Journal of Physics. D: Applied Physics, 36,1850-1857 (2003).

16. C. R. Petersen, U. Møller, I. Kubat, B. Zhou, , S. Dupont, J. Ramsay, T. Benson, S. Sujecki, N. AbdelMoneim, Z. Tang, D. Furniss, A. Seddon and O. Bang, "Mid-infrared supercontinuum covering the 1.4$13.3 \mu \mathrm{m}$ molecular fingerprint region using ultra-high NA chalcogenide step-index fibre", Nature Photonics, 8 (11),830-834, 2014.

17. N. Carlie, N. Anheier, H. Qiao, B. Bernacki, M. Phillipes, L. Petit, J. Musgraves and K. Richardson, "Measurement of the refractive index dispersion of $\mathrm{As}_{2} \mathrm{Se}_{3}$ bulk glass and thin films prior to and after laser irradiation and annealing using prism coupling in the near- and mid-infrared spectral range", Rev. Sci. Instrum., 82, 053103, 2011.

18. H. Qiao, N. Anheier, J. Musgraves, K. Richardson and D. Hewak, "Measurement of Chalcogenide Glass Optical Dispersion Using a Mid- Infrared Prism Coupler", Proc. of SPIE, Vol. 8016(80160F), 1-10, 2011.

19. D. Tentori and R. Lerma, "Refractometry by minimum deviation: Accuracy analysis", Optical Engineering, 29(2), 160-168, 1990.

20. R. Swanepoel, "Determining refractive index and thickness of thin films from wavelength measurements only", Optical Society of America, 2(8), 1339-1343,1985.

21. C. Corrales, J. Ramírez-Malo, J. Fernández-Peña, P. Villares, R. Swanepoel and E. Márquez (1995) "Determining the refractive index and average thickness of AsSe semiconducting glass films from wavelength measurement only", Applied Optics, 34(34), 7907-7913,1995.

22. M. S. Maklad, R. K. Mohr, R. E. Howard, P. B. Macedo and C. T. Moynihan, "Multiphonon absorption in $\mathrm{As}_{2} \mathrm{~S}_{3}-\mathrm{As}_{2} \mathrm{Se}_{3}$ glasses," Solid State Commun. 15(5), 855-858, 1974. 